\title{
Incidencia de las Políticas Educativas implementadas por Ministerio de Educación de Guatemala
}

\section{Incidence of Educational Policies implemented by the Ministry of Education of Guatemala}

Recibido 15/08/2021

\author{
Yadira Abigail Ishlaj Conde \\ Centro Universitario de Sur Oriente \\ Universidad de San Carlos de Guatemala \\ yadirabic@hotmail.com \\ https://orcid.org/0000-0002-0371-1348
}

Aceptado 19/10/2021

Ishlaj Conde, Y. A. (2021). Incidencia de las Políticas Educativas implementadas por Ministerio de Educación de Guatemala. Revista Guatemalteca De Educación Superior, 5(1). 64-76

https://doi.org/10.46954/revistages.v5i1.74

\section{Resumen}

OBJETIVO: realizar un análisis de la incidencia de las Políticas Educativas de los períodos de gobierno del 2008 al 2020. MÉTODO: cualitativo, investigación documental. RESULTADOS: se logró determinar que las políticas educativas de los años 2008 hasta el 2020 enfatizan en el mejoramiento de la calidad de educación en las aulas y la gestión educativa; la ampliación de la cobertura de centros educativos que atienden niños y jóvenes de las áreas urbana y rural del país. Los guatemaltecos necesitamos un sistema educativo operante que permita cambios de patrones donde se formen ciudadanos capaces de activar la economía y minimizar la brecha entre países de desarrollo sustancial. CONCLUSIÓN: el análisis de las políticas educativas implementadas en los años 2008 hasta el 2020 muestran esfuerzos en actualizar las prácticas administrativas, innovar la 
entrega docente; por medio de la atención en cuatro escenarios principales que debe priorizar la planificación estratégica del Estado de Guatemala a través de su política pública para promover acciones sociopolíticas, educación obligatoria, profesionalización del magisterio y pactos de colaboración con autoridades municipales. Este estudio establece una investigación para determinar que las políticas de estos años enfatizan en el mejoramiento de la educación.

\section{Abstract}

OBJECTIVE: To carry out an analysis of the incidence of the Educational Policies of the government periods from 2008 to 2020. METHOD: qualitative, documentary research. RESULTS: It was possible to determine that the educational policies of the years 2008 to 2020 emphasize the improvement of the quality of education in the classrooms and educational management; expanding the coverage of educational centers that serve children and young people in urban and rural areas of the country. We Guatemalans need a functioning educational system that allows changes in patterns where citizens capable of activating the economy and minimizing the gap between countries with substantial development are formed. CONCLUSION: The analysis of the educational policies implemented in the years 2008 to 2020 show efforts to update administrative practices, innovate teaching delivery; Through attention to four main scenarios that must prioritize the strategic planning of the State of Guatemala through its public policy to promote sociopolitical actions, compulsory education, professionalization of the teaching profession and collaboration agreements with municipal authorities. This study establishes an investigation to determine that the policies of these years emphasize the improvement of education.

\section{Introducción}

El Ministerio de Educación de Guatemala depende de un prepuesto asignado en forma anual, mismo que no es suficiente para solventar la demanda en cobertura, retención de matrícula

\section{Palabras clave:}

políticas educativas, ministerio de educación, plan estratégico, incidencia, derecho.

\section{Keywords:}

educational policies, ministry of education, strategic plan, advocacy, law. 
e implementación de mobiliario e infraestructura en centros educativos públicos de las áreas rurales y urbanas; pero a pesar de ello las Políticas Educativas y los ejes de intervención por medio del plan estratégico anual del Ministerio de Educación (MINEDUC) permiten la intervención por medio de programas del Viceministerio Técnico de Educación, Viceministerio de Educación Bilingüe e intercultural y Viceministerio Extraescolar y alternativa.

\section{Contenido}

En Guatemala por mandato constitucional es obligación del Ministerio de Educación el brindar educación laica, gratuita y obligatoria; como respuesta al cumplimiento de lo estipulado a partir de la firma de los Acuerdos de Paz Firme y Duradera el 29 de diciembre de 1996 por medio de la propuesta de Reforma Educativa; misma que conlleva determinar el rumbo de la educación pública y privada del pais.

En las Políticas Educativas de los últimos tres gobiernos en turno se puede asegurar que las prioridades en el tema educativo estuvieron enfocadas al aseguramiento de la calidad educativa, el fortalecimiento y profesionalización del recurso humano en la docencia; la dotación de mobiliario y equipo tecnológico, infraestructura, gestión administrativa y fortalecimiento de la Educación Bilingüe Intercultural en atención a los cuatro pueblos que conforman la población guatemalteca: Xinca, Garífuna, Maya y Ladino.

\section{Políticas Educativas 2008-2012 en Guatemala}

En el periodo de gobierno del Presidente Álvaro Colom Caballeros las políticas educativas abordadas fueron: ampliación de cobertura del sistema educativo, mejorar la calidad educativa, recursos humanos e infraestructura educativa, ampliación y fortalecimiento de los programas de alfabetización con modalidad bilingüe y con el enfoque de equidad de género, fortalecimiento de la educación bilingüe intercultural EBI y formación de recurso humano a nivel superior (Secretaria de 
Planificación y Programación de la Presidencia IV Informe Presidencial y memoria de Labores 2011:45) .

En este periodo de gobierno se prioriza la política Avanzar hacia una Educación de Calidad en apego al derecho a la educación, considerado no sólo la asistencia a un centro educativo; sino tener acceso a una educación respetando en forma igualitaria las condiciones sociales de: riqueza - pobreza, mujer u hombre, raza - religión (UNESCO, 2007, p. 11).

Todos deben de gozar de una educación pertinente y relevante con las condiciones que les permitan ejercer ciudadanía en los años venideros y desempeñarse con aptitudes y actitudes ante los desafíos de un empleo en un contexto local, regional y nacional; teniendo como punto de partida su cultura y la convivencia solidaria comunitaria (Constitución Política de Guatemala, 1995).

La Política Educativa de ampliar la cobertura educativa se resumió al postulado de la inclusión de niños y niñas de extrema pobreza y de condiciones vulnerables; en cumplimiento al mandato constitucional de Guatemala y al cumplimiento de los Acuerdos de Paz que establece la obligatoriedad de la educación inicial, la educación pre primaria, primaria y educación media en sus niveles: Básico y Diversificado; con un carácter gratuito por lo que el plan 2008 - 2012 estima la ampliación de los servicios educativos en todos sus nieles y es el Presidente Álvaro Colon Caballeros quien implementa para esto los programas de apoyo constituidos por aportes para refacción escolar, útiles escolares, valija didáctica y fondo de gratuidad de la educación para reparaciones y mantenimiento de edificios escolares.

La Política Educativa de Equidad promulgo el acceso de la mujer guatemalteca a la educación en todos los niveles que históricamente había sido privada de ese derecho por estereotipos heredados de patrones patriarcales en Guatemala, además incluyo la participación y reconocimiento de poblaciones indígenas quienes habían permanecido al margen del derecho universal a la educación; todo esto conlleva garantizar como Ministerio de Educación la prestación del servicio en todas las regiones del país, 
enfatizando la educación bilingüe e intercultural. La política de equidad recibió apoyo gubernamental para su implementación por medio del programa de Transferencias Condicionadas en las que familiar de área rural y con preeminencia indígenas recibían un aporte económico mensual con el condicionamiento que los niños y jóvenes estuvieran inscritos en los centros educativos de su comunidad.

La Política Educativa de Educación Bilingüe fortaleció la identidad lingüística y cultural de todos los pueblos que integran Guatemala y que constituyen una riqueza para el país. En este periodo de gobierno se incrementó el presupuesto al Vicedespacho de Educación Bilingüe Intercultural y por primera vez se implementaron mesas de diálogo para el respeto a la cosmovisión del pueblo maya; implementando textos, materiales y recursos de enseñanza por pueblo (Garífuna, Xinca y Maya), además se incrementó la contratación de maestros bilingües, mejorando las condiciones laborales establecidas en la Ley de Generalización de la Educación Bilingüe Intercultural en Guatemala (MINEDUC, 2004, p. 23).

Toda la logística que conlleva la implantación de las políticas educativas, en este caso la de Educación Bilingüe incurre en acciones ineludibles como la implementación de ejes, para este caso el primer eje contempla: la ciudanía multicultural en respuesta a la identidad local, el segundo eje el contexto de la ciudadanía guatemalteca y el tercer eje vinculado a la ciudadanía centroamericana y cosmopolita.

La política de Modelo de Gestión implementada enfatizó en establecer mecanismos de eficacia, transparencia y eficiencia que se garantizaron por medio de los principios de participación, descentralización y pertinencia que caracterizaron cada uno de los centros educativos del país y las instancias administrativas de cada una de las jurisdicciones. 
En este período las políticas educativas que se trazaron conjuntamente con instituciones nacionales e internacionales, las Metas del Milenio y las Metas 20-21 aportan una iniciativa que dará respuesta al complejo escenario educativo que necesita atender problemas sociales, culturales y de pertinencia económica laboral (Consejo Nacional de Educación (2010) Políticas Educativas: 2-3).

La Políticas Educativas implementadas en este gobernó del General Otto Pérez Molina fueron aprobadas según Acuerdo Ministerial número 3409-2011 de fecha 30 de noviembre de 2011 donde la primera política hace referencia a la cobertura que garantiza el acceso, permanencia y egreso efectivo de la niñez y la juventud sin discriminación a todos los niveles educativos y subsistemas escolares y extraescolares. Dentro de esta política de cobertura es imperante hacer mención del incremento en todos los niveles, así como garantizar las condiciones que permitan la permanencia y el egreso de los estudiantes acción que por primera vez no queda reducida a la inscripción como indicador de cobertura, sino estima la permanencia y la finalización o egreso de los estudiantes. Además, cabe mencionar que se enriquece esta política cuando amplia programas extraescolares.

La segunda Política Educativa es la de Calidad, en la que la meta es mejorar el proceso educativo por medio del aseguramiento que todos los alumnos reciban una educación pertinente y relevante; esto por medio de enfoques curriculares en temas relacionados a la tecnología; pero a la vez se implementaron evaluaciones para garantizar la calidad educativa. Bolaños y Santos (2015) asegura que se deben hacer evidente los esfuerzos por elevar sustancialmente la calidad educativa con la implantación del currículo en el aula y los resultados de las evaluaciones a graduandos como reflejo de alcanzar los perfiles terminales establecidos en el Currículo Nacional Base. 
La política Modelo de Gestión se enfocó en fortalecer sistemáticamente los mecanismos de efectividad y transparencia en el sistema educativo, esta política sistematizó el proceso de información educativa y fortaleció la gestión para alcanzar la efectividad del proceso educativo que conllevó acciones in situ de cuantificar recursos y beneficiarios permitiendo garantizar la transparencia en la rendición de cuentas; una variante y acción significativa fue el establecer un sistema de remozamiento, mantenimiento y construcción de la planta física de los centros educativos de todo el país, donde por primera vez en diez años se intervino a nivel de centro educativo priorizando techos, baños, sistema eléctrico y de agua potable.

La cuarta Política Educativa: Recurso Humano se concretizó al fortalecimiento de la formación, evaluación y gestión del recurso humano del sistema educativo nacional por medio de la cual se trabajó en capacitaciones de formación y actualización del docente hasta alcanzar un desempeño efectivo.

La Política Educativa Bilingüe Multicultural e Intercultural es la quinta de este periodo gobierno que fortaleció por medio de programas la participación entre los pueblos y formas culturales de vida, implementando mallas curriculares que respeten las características socioculturales de cada pueblo; además garantizó la cobertura de la Educación Bilingüe multicultural e intercultural. Algo importante que vale la pena resaltar es el sistema de acompañamiento técnico de aula especifico de la EBMI.

La sexta Política Educativa es el aumento a la Inversión Educativa por medio de la asignación presupuestaria a la educación hasta alcanzar lo que establece el artículo 102 de la Ley de Educación Nacional el $7 \%$ del producto bruto interno.

La Política Educativa de Equidad, como la séptima implementada está vinculada a promover una educación con calidad que demanda que las personas que conforman los pueblos que integran Guatemala sean respetados y reconocidos desde su contexto y el mundo actual. Esta política asegura que el Ministerio de Educación permita el acceso a la educación 
integral con equidad y en igualdad de oportunidades; reduce el fracaso escolar en los grupos más vulnerables. Por último, la octava Política Educativa es el Fortalecimiento Institucional y Descentralización que fomenta la participación de la comunidad educativa desde el ámbito local que fortalece y garantiza la calidad, cobertura y permanencia de los estudiantes mediante la promoción del respeto social, cultural y lingüístico en todos los niveles educativos, modalidades y visión.

\section{Políticas Educativas 2016 - 2020 en Guatemala}

El Ministerio de Educación en cumplimiento al Plan Estratégico 2016-2020 brinda libre acceso a la educación en todos sus niveles; por medio de las acciones que conllevan implementar las políticas educativas que ayuden a mejorar los servicios educativos. Las autoridades educativas brindan importancia el fortalecimiento de sus dependencias en los ámbitos departamental y local; por medio de la gestión administrativa desconcentrada y descentralizada.

Los diseños de la planificación estratégica se basaron en la identificación de las necesidades en las áreas de cobertura para incrementar la tasa escolar, remozar infraestructura, implementar tecnología, calidad educativa en el aula, modelo de gestión, entre otros (MINEDUC, Plan Estratégico 2016-2020: 06-07).

En el gobierno del presidente Licenciado Jimmy Morales Cabrera siguen vigentes las Políticas Educativas establecidas en el Acuerdo Gubernativo número 3409-2011 y que el Señor Ministro de Educación Dr. Oscar Hugo López Rivas ratifica su cumplimiento bajo los principios de acción: transparencia, inclusión, dialogo y participación social, pertinencia lingüística y cultural, Multiculturalidad e interculturalidad, educación como un derecho y equidad e igualdad (MINEDUC, Plan Estratégico 2016-2020: 06-07).

El plan estratégico que da cumplimiento a las políticas establecidas está organizado en ejes prioritarios siendo el primero el de Cobertura que afirma que la niñez y juventud tiene 
sin distinción de ninguna clase acceso a programas escolares y extraescolares con pertinencia lingüística y cultural. Eje que se verificará al culminar el 2020 con el incremento de la cobertura de la preprimaria de 47.3 al $59.3 \%$, en primaria del 82 al $88 \%$, en ciclo básico del 46 al $50 \%$ y en diversificado del $24 \%$ al $28 \%$ (MINEDUC, Plan Estratégico 2016-2020:8) indicadores puntuales que podrán evidenciar el cumplimiento de las políticas y los ejes prioritarios del referido plan.

El eje Calidad, Equidad e Inclusión asegura la participación de la niñez y juventud en todos los programas en los que se manifiesta la entrega de calidad, equidad y pertinencia lingüística y con respeto a su cultura materna. Para el 2020 se podrá medir el alcance de este eje con el indicador de haber incrementado en un $7 \%$ los servicios de atención a la niñez y juventud con capacidades especiales; así como la implementación de modelos innovadores en todos los niveles del sistema educativo nacional (MINEDUC, Plan Estratégico 2016-2020: 12).

El eje Modalidades Diversas de entrega Escolar y Extraescolar refiere a que los niños, jóvenes y adultos serán atendido dentro del sistema nacional de educación con pertinencia lingüística y cultural; lográndose por medio de las líneas de acción que intervienen en procesos de evaluación pertinente de las modalidades de entrega educativa e implementar esas buenas prácticas en los diferentes ámbitos. Además, la alternancia educativa podrá hacerinclusión de gruposno atendidosfacilitando su acceso a la educación y minimizando barreras de diversa índole; por último, la creación de PRONEA (Programa Nacional de Educación Alternativa) es una modalidad de educación que permite integrar a la población migrante y acreditar y certificar sus estudios de primaria, básico y diversificado; pero también facilita que guatemaltecos sin la oportunidad ahora puedan completar sus estudios con un fácil acceso a un programa que facilita sus estudios por medio de intervenciones en On Line y con tutores que acompañan el proceso en horarios flexibles.

En el eje Espacios Dignos y Saludables para el Aprendizaje se está identificando centros educativos con necesidades de reparaciones y remozamientos para convertirlos en 
espacios dignos y saludables a través de una intervención gubernamental, con apoyo de organizaciones internacionales y amigos cooperantes de la educación. Es importante también resaltar que en el ejercicio de la administración pública el Ministerio de Educación se encuentra legalizando los terrenos de centros educativos públicos para poder hacer inversiones en infraestructura que permitan la construcción de aulas, baterías de servicios sanitarios, cocinas y áreas recreativas escolares.

Por último el eje Gestión Institucional y Participativo donde como ministerio se realizan acciones desconcentradas donde se fortalece la vida democrática del país, a través de la organización de Padres de Familia que ayudan en la administración de fondos proporcionados por medio de la modalidad de transferencias para ejecutar los Programas de Apoyo, permitiendo este modelo de gestión que se establezca en cada Dirección Departamental de Educación capacidades administrativas y financieras que permitan dar mejor atención a cada centro educativo de su jurisdicción.

\section{Conclusión}

Las Políticas Educativas de los últimos tres períodos de gobiernos responden a lo establecido en el Acuerdo Ministerial Número 3409-2011 donde se aprueban las Políticas Educativa: Cobertura, Calidad, Educación Bilingüe Multicultural e Intercultural, Aumento de la Inversión Educativa, Equidad y Fortalecimiento Institucional y Descentralización. La Política Educativa de Cobertura en concordancia con los objetivos de desarrollo del milenio prioriza la ampliación de cobertura nacional con equidad, creando estrategias para el aumento de la matrícula escolar.

Mejorar la calidad educativa como política siempre ha sido prioridad en las últimas tres administraciones gubernamentales y ministeriales; esta política educativa ha apuntalado la implementación de programas y acciones innovadoras para avanzar en el logro de una educación de calidad, adaptada al mundo cambiante en el que vivimos. 
En el tema de Educación Bilingüe Multicultural e Intercultural se reconoce en los Acuerdo de Paz, el diseño de Reforma Educativa, La Constitución Política de la República de Guatemala, la Ley de Educación Nacional y el Acuerdo Gubernativo 222004 Generalización de la educación Bilingüe, Multilingüe e Intercultural, que esta política debe buscar el desarrollo integral de los estudiantes mediante procesos de aprendizaje contextualizados cultural y lingüísticamente.

En los temas de Inversión Educativa, Equidad y Gestión Institucional los tres periodos dan prioridad por medio de acciones que conllevan la creación de centros educativos, dotación de mobiliario a centros educativos, evaluación e intervención de escuelas en conjunto con cooperativas, ONGs, Municipalidades, Consejos Departamentales de Desarrollo, donantes internacionales para la reparación, ampliación y construcción de centros educativos

Po lo que las Políticas Públicas como las Políticas Educativas implementadas por el Ministerio de Educación de la República de Guatemala reiterativas en los últimos tres periodos de gobierno son: Ampliación de la cobertura, Mejoramiento de la Calidad, Participación Comunitaria, Educación Bilingüe Intercultural, Descentralización Administrativa, Modelo de Gestión, Incremento a la Inversión Educativa y consolidación de un Sistema Democrático.

\section{Referencias}

Bolaños, V. y Santos, J. (2015). Informe de los resultados de la evaluación de graduandos 2014. Guatemala: Dirección General de Evaluación e Investigación Educativa, Ministerio de Educación de Guatemala. https://www. mineduc.gob.gt/digeduca/documents/informes/ Informe_Graduandos2014.pdf

Consejo Nacional de Educación (2010). Políticas Educativas.

Recuperado de: http://www.mineduc.gob.gt/ estadistica/2011/data/Politica/Politicas_Educativas_ CNE.pdf 
Constitución Política de la República de Guatemala. (1985). https://www.cijc.org/es/NuestrasConstituciones/ GUATEMALA-Constitucion.pdf

Ley de Generalización de la Educación Bilingüe, Multilingüe e Intercultural, Acuerdo Gubernativo 22-2004.(2004). Guatemala. https://www.congreso.gob.gt/assets/ uploads/info_legislativo/dictamen/1058.pdf

Ministerio de Educación (2006). Lineamientos educativos para la diversidad cultural y lingüística de Guatemala (Guatemala). https://www.mineduc.gob.gt/DIGEBI/ documents/modeloEBI.pdf

Ministerio de Educación (2008). Políticas Educativas Ministeriales 2008- 2011. Guatemala. http://infopublica. mineduc.gob.gt/mineduc/images/5/58/DIDEFI_POL_ EDU_ACUERDO_INCISO5B_2012_VERSION1.pdf

Ministerio de Educación (2002) Programa de Desarrollo Profesional. Culturas e Idiomas de Guatemala. (Guatemala, MINEDUC - DICADE)

Ministerio de Educación (2012). Plan de Implementación Estratégica de Educación 2012-2016. Guatemala: Recuperado de: http://www.mineduc. gob.gt/portal/ contenido/menu_lateral/quienes_somos/politicas_ educativas/pdf/plan\%20de\%20implementacion\%20 estrategica\%20de\%20educacion\%202012-016.pdf

Ministerio de Educación (2012). Estrategia para una Educación de Calidad para la niñez y la juventud guatemalteca. Recuperado de: http://www.mineduc. gob.gt/PORTAL/ contenido/anuncios/estrategiaCalidadEducativa/ documents/Presentacion\%20Estrategia\%20para\%20 una\%20educaci\%C3\%B3n\%20 de\%20calidad.pdf

UNESCO (2007). Educación de calidad para todos: un asunto de derechos humanos. Buenos Aires, Argentina: Recuperado de: http://unesdoc.unesco. org/ images/0015/001502/150272s.pdf 


\section{Sobre la autora}

Yadira Abigail Ishlaj Conde de González, Maestra en Docencia Universitaria con especialidad en Evaluación Educativa. Licenciada en Pedagogía y Administración Educativa. Profesora de Enseñanza Media en Pedagogía y Ciencias de la Educación. Diplomado Internacional en Cultura de la Investigación On line modalidad Ciencias Sociales. Diplomado en Informática con énfasis en Administración. Curso de Actualización de Investigación Científica, modalidad e - learning. Diplomado Calidad en la Educación Superior. Diplomado de Actualización Docente y Aula Virtual. Diplomado en línea de actualización en herramientas digitales DIGITAL "TOOLS FOR EDUCATION". Docente Investigadora Centro Universitario de Sur Oriente Departamento de Postgrado y Carrera de Pedagogía. Coordinadora de Capacitación del Departamento de Investigación CUNSURORI, Jalapa.

\section{Conflicto de intereses}

Declara no tener ningún conflicto de intereses.

\section{Declaración de consentimiento informado}

El estudio se realizó con fines educativos, respetando el Código de ética y buenas prácticas editoriales de publicación.

\section{Derechos de uso}

Copyright@ 2021 por Yadira Abigail Ishlaj Conde

Este texto está protegido por la Licencia Creative Commons Atribución 4.0 Internacional.

Usted es libre para compartir, copiar y redistribuir el material en cualquier medio o formato y adaptar el documento, remezclar, transformar y crear a partir del material para cualquier propósito, incluso comercialmente, siempre que cumpla la condición de atribución: usted debe reconocer el crédito de una obra de manera adecuada, proporcionar un enlace a la licencia, e indicar si se han realizado cambios. Puede hacerlo en cualquier forma razonable, pero no de forma tal que sugiera que tiene el apoyo del licenciante o lo recibe por el uso que hace. 